\title{
Ring chromosome 1 syndrome
}

INSERM

\section{Source}

INSERM. (1999). Orphanet: an online rare disease and orphan drug data base. Ring chromosome 1 syndrome. ORPHA:1437

Ring chromosome 1 syndrome is an autosomal anomaly characterized by variable clinical features, most commonly including significant intrauterine and postnatal growth failure, developmental delay, intellectual disability, microcephaly, and dysmorphic facial features. Some less frequent clinical features are dysgenesis of corpus callosum, atrial septal defect, rocker bottom feet and clinodactyly. 